\title{
COMTRANS ALGEBRAS AND THEIR PHYSICAL APPLICATIONS
}

\author{
JONATHAN SMITH \\ Department of Mathematics, Iowa State University \\ Ames, Iowa 50011, U.S.A.
}

1. Introduction. Comtrans algebras were introduced [15] in answer to a problem from differential geometry [3, Problem X.3.9], [10, p. 16]: finding the algebraic structure in the tangent bundle corresponding to the coordinate $n$-ary loop of an $(n+1)$-web $[4, \S 3.7]$. The algebraic structure consists of a system of comtrans algebras, interlaced with some of the "W-algebras" (now known as Akivis algebras) that had been introduced earlier by Akivis in correspondence with the coordinate binary loops of 3 -webs [1], [3, §IX.6], [6]. A Lie group determines a special kind of 3-web, a group web [3, Defn. X.6.2], and the corresponding Akivis algebra in this case is a Lie algebra. In this sense comtrans algebras are general ternary analogues of Lie algebras. To some extent the theory of comtrans algebras is modelled on, and may subsume, the theory of Lie algebras.

The purpose of the current paper is to provide a brief survey of the present (early) state of the theory of comtrans algebras, placing particular emphasis on physical applications. Basic definitions are given in Section 2. Section 3 is devoted to presenting a number of examples of comtrans algebras, namely on Lie algebras, spaces with bilinear forms, and spaces of rectangular matrices. Physical interest here focuses on the comtrans algebra structure on Minkowski space-time that extends the vector triple product on $\mathbb{R}^{3}$. The fourth section describes the transposed comtrans algebra that is (term) equivalent to a given comtrans algebra. Representation theory of comtrans algebras is discussed briefly in the fifth section, in terms of the universal enveloping algebra. The final section presents a new example of comtrans algebras, on spaces of Hermitian operators. The primary motivation and application here is quantum mechanics. Formulating quantum mechanics in

1991 Mathematics Subject Classification: 17D99, 53A60, 81R05.

The paper is in final form and no version of it will be published elsewhere. 
terms of comtrans algebras, the choice of sign of Planck's constant is seen to represent no more than a choice between two (term) equivalent presentations of an algebraic theory.

2. Comtrans algebras. Let $R$ be a commutative ring with 1 . A comtrans algebra over $R$ is a unital $R$-module $E$ equipped with a trilinear operation

$$
[,,]: E^{3} \rightarrow E ; \quad(x, y, z) \mapsto[x, y, z]
$$

called the commutator and a trilinear operation

$$
\langle,,\rangle: E^{3} \rightarrow E ; \quad(x, y, z) \mapsto\langle x, y, z\rangle
$$

called the translator. The commutator is left alternative, in the sense that

$$
[x, x, z]=0 .
$$

The translator satisfies the Jacobi identity

$$
\langle x, y, z\rangle+\langle y, z, x\rangle+\langle z, x, y\rangle=0 .
$$

Finally, the commutator and translator together satisfy the comtrans identity

$$
[x, y, x]=\langle x, y, x\rangle \text {. }
$$

For a fixed ring $R$, the class $R-C T$ of comtrans algebras forms a variety in the sense of universal algebra. The notation $R-\underline{C T}$ (or just $\underline{C T}$ if $R$ is implicit) is also used for the category whose objects are comtrans algebras over $R$ and whose morphisms are homomorphisms between them. As a variety, $R-\underline{\underline{C T}}$ is a Mal'cev variety (in the sense of [14]), and thus has well-behaved centrality and cohomology. A comtrans algebra is said to be abelian if its commutators and translators are identically zero. Ideals in a comtrans algebra $E$ are the congruence classes of 0 . A comtrans algebra is said to be simple if it is non-abelian, but has no proper non-trivial ideals. These concepts are all analogous to their counterparts in the theory of Lie algebras.

Given a trilinear operation

$$
E^{3} \rightarrow E ; \quad(x, y, z) \mapsto x y z
$$

on a unital $R$-module $E$, the commutator of the operation is

$$
[x, y, z]=x y z-y x z,
$$

and the translator of the operation is

$$
\langle x, y, z\rangle=x y z-y z x .
$$

Under these operations, $E$ forms a comtrans algebra. This is analogous to the construction of a Lie algebra with commutator

$$
[x, y]=x y-y x
$$

from a bilinear associative operation $(x, y) \mapsto x y$. If 6 is invertible in the ring $R$, each comtrans algebra over $R$ is obtainable from the commutator and translator of a suitably defined trilinear operation (2.6) [15, Prop. 3.3]. 
3. Examples of comtrans algebras. Given a Lie algebra $L$ over the ring $R$, a comtrans algebra $C T(L)$ is obtained by taking the commutator and translator to be the repeated Lie algebra commutator:

$$
[x, y, z]=\langle x, y, z\rangle=[[x, y], z] .
$$

In this case, the Jacobi identity in the comtrans algebra $C T(L)$ corresponds exactly to the Jacobi identity in the Lie algebra $L$. If the Lie algebra $L$ is abelian, then so is the comtrans algebra $C T(L)$. Simple Lie algebras provide the first examples of simple comtrans algebras, and these comtrans algebras essentially capture all the structure of the Lie algebras:

(3.2) Theorem [11, Th. 3.2]. A Lie algebra L is simple if and only if the corresponding comtrans algebra $C T(L)$ is simple.

The most elementary Lie algebra of physical significance is the 3-dimensional real Lie algebra $\left(\mathbb{R}^{3}, \times\right)$ given by the "cross" or "vector" product of vectors. The corresponding comtrans algebra $C T\left(\mathbb{R}^{3}, \times\right)$ is well known; its equal commutators and translators are the "vector triple products"

$$
(\underline{x} \times \underline{y}) \times \underline{z}=(\underline{x} \cdot \underline{z}) \underline{y}-(\underline{y} \cdot \underline{z}) \underline{x} .
$$

The vector product is a notoriously "accidential", exceptional phenomenon, and there are no analogous Lie algebras on other normed spaces. However, the right hand side of the identity (3.3) suggests a way of putting a comtrans algebra structure $C T(E, \beta)$ on a formed module $(E, \beta)$, a unital module $E$ over the commutative ring $R$ equipped with a bilinear form $\beta: E \times E \rightarrow R$. The algebra $C T(E, \beta)$ has commutator

$$
[x, y, z]=y \beta(x, z)-x \beta(y, z)
$$

and translator

$$
\langle x, y, z\rangle=y \beta(z, x)-x \beta(y, z) .
$$

Except for the trivial cases, the comtrans algebra $C T(E, \beta)$ encodes all the structure in $(E, \beta)$. Define a formed space to be a formed module $(E, \beta)$ whose underlying module is free of rank more than 1 . Recall the definition of the radical:

$$
\operatorname{Rad} \beta=\{x \in E \mid \forall y \in E, \beta(x, y)=\beta(y, x)=0\} .
$$

One then has the following

(3.7) Theorem [12]. Let $(E, \beta)$ be a formed space, with corresponding comtrans algebra $C T(E, \beta)$. Then:

(a) $\operatorname{Rad} \beta=\{x \in E \mid \forall y, z \in E,\langle z, y, x\rangle=0\}$;

(b) the automorphism groups of $(E, \beta)$ and of $C T(E, \beta)$ coincide;

(c) $C T(E, \beta)$ is simple if and only if $\operatorname{Rad} \beta=\{0\}$ and $R$ is a field.

The theorem shows that the Lorentz metric on 4-dimensional real space-time provides a simple comtrans algebra that extends the 3-dimensional vector triple product comtrans algebra. Moreover, the Lorentz group is the automorphism 
group of this 4-dimensional comtrans algebra. In general, comtrans algebras provide the most compact and efficient algebraisation of bilinear forms. Other algebraisations, such as Jordan algebras and Clifford algebras, require an expansion of the underlying module. The underlying module of a Clifford algebra, for instance, is exponentially large compared to the underlying module of the space carrying the quadratic form.

The comtrans algebras $C T(E, \beta)$ of formed spaces $(E, \beta)$ whose underlying module has finite rank are special cases of comtrans algebra structure that is carried by the set of rectangular matrices of a given size. Let $R_{m}^{n}$ denote the $R$-module of $(m \times n)$-matrices over the commutative ring $R$. Fix $A$ in $R_{n}^{n}$ and $B$ in $R_{m}^{m}$. Set $E=R_{m}^{n}$. Then the commutator (2.7) and translator (2.8) of the trilinear operation

$$
(X, Y, Z) \mapsto X A Y^{T} B Z
$$

on $E$ give a comtrans algebra $C T(A, B)$. Define

$$
R(A): E \rightarrow E ; \quad X \mapsto X A
$$

and

$$
L(B): E \rightarrow E ; \quad X \mapsto B X .
$$

The simple comtrans algebras $C T(A, B)$ are then described as follows.

(3.11) Theorem [11, Th. 4.7]. A comtrans algebra $C T(A, B)$ is simple if and only if $R$ is a field, $n m>1$, and $\operatorname{Ker} R(A) \cap \operatorname{Ker} R\left(A^{T}\right)=\{0\}=\operatorname{Ker} L(B) \cap$ $\operatorname{Ker} L\left(B^{T}\right)$.

4. Transposed algebras. Given a comtrans algebra $E$ with commutator $[x, y, z]$ and translator $\langle x, y, z\rangle$, a new comtrans algebra $E^{\tau}$, called the transposed algebra, is defined on the same underlying module $E$, with commutator

$$
[x, y, z]^{\tau}=[z, y, x]+\langle y, z, x\rangle
$$

and translator

$$
\langle x, y, z\rangle^{\tau}=-\langle x, z, y\rangle \text {. }
$$

Note that $E^{\tau \tau}=-E$, so that the algebras $E$ and $E^{\tau}$ are (term) equivalent in the sense of universal algebra [16, p. 13]. This implies that $E$ and $E^{\tau}$ have the same ideals, so that $E$ is simple if and only if $E^{\tau}$ is. A comtrans algebra $E$ is said to be symmetric if it is equal to its transpose, and antisymmetric if $[x, y, z]^{\tau}=-[x, y, z]$ and $\langle x, y, z\rangle^{\tau}=-\langle x, y, z\rangle$. If 2 is invertible in the ring $R$, each comtrans algebra $E$ over $R$ decomposes into the sum of a symmetric part $\left(E+E^{\tau}\right) / 2$, e.g. with commutator

$$
\left([x, y, z]+[x, y, z]^{\tau}\right) / 2
$$


and an antisymmetric part $\left(E-E^{\tau}\right) / 2$. Matrix transposition gives an isomorphism between the comtrans algebras $C T(A, B)^{\tau}$ and $C T\left(B^{T}, A^{T}\right)$ defined from (3.8).

Transposition provides a convenient way of recognizing which comtrans algebras are the comtrans algebras $C T(E, \beta)$ of a formed space:

(4.4) TheOREm [12, Th. 4.1]. Let $E$ be a comtrans algebra whose underlying module is free of rank at least 3 . Then there is a bilinear form $\beta$ on $E$ such that $E=C T(E, \beta)^{\tau}$ if and only if, for all $x, y, z$ in $E$, one has $[x, y, z] \in z R$ and $\langle x, y, z\rangle \in x R+z R$.

An interesting example shows the necessity of the rank condition in Theorem 4.4. Let $(H, \alpha)$ be a non-degenerate 2-dimensional symplectic space (or "hyperbolic plane" in the sense of [7, Def. II.9.7]) over a field $R$. Then

$$
[x, y, z]=-z \alpha(y, x)
$$

and

$$
\langle x, y, z\rangle=x \alpha(z, y)
$$

define a comtrans algebra on $H$. Nevertheless, this comtrans algebra is not the transpose of the algebra $C T(H, \beta)$ for any bilinear form $\beta$ on $H$ [12, Ex. 4.2].

5. Representation theory. Fix a commutative ring $R$, and consider the Mal'cev variety $\underline{C T}$ of comtrans algebras over $R$. As a category, $\underline{C T}$ is complete and cocomplete $[5,32.14]$. For a comtrans algebra $E$ over $R$, define $E[X]$ to be the coproduct in $\underline{\underline{C T}}$ of $E$ with the free $\underline{\underline{C T}}$-algebra on a single generator $X$. Identify $E$ with its image in $E[X]$. For $x, y$ in $E$, there are module endomorphisms

$$
\begin{array}{rll}
K(x, y): E[X] \rightarrow E[X] ; & z \mapsto[z, x, y], & \\
R(x, y): E[X] \rightarrow E[X] ; & z \mapsto\langle z, x, y\rangle, \quad \text { and } \\
L(x, y): E[X] \rightarrow E[X] ; & z \mapsto\langle y, x, z\rangle .
\end{array}
$$

The universal enveloping algebra $U(E)$ is defined to be the subalgebra of the $R$-module endomorphism ring $\operatorname{End}_{R} E[X]$ generated by

$$
\{K(x, y), R(x, y), L(x, y) \mid x, y \in E\} .
$$

There are universal-algebraic ways of determining what a module over the comtrans algebra $E$ should be [14, p. 9], but it turns out that these modules are equivalent to right modules over the universal enveloping algebra $U(E)$ [13, Th. 3.10]. As an elementary illustration, consider the centre $Z(E)$ of the comtrans algebra $E$. Universal-algebraically, the centre is defined as the congruence class of 0 under the centre congruence $\zeta(E)[14$, p. 42$]$. However, $E$ is a submodule of the right $U(E)$-module $E[X]$, and $Z(E)$ is then just the submodule of $E$ fixed by $U(E)$ [13, Prop. 2.3]. If $R$ is a field, then the universal enveloping algebra is described by 
(5.5) THEOREM [13, Th. 4.5]. Let $E$ be a comtrans algebra over a field $R$. Let $V=(E \wedge E) \oplus(E \otimes E) \oplus(E \otimes E)$. Then the universal enveloping algebra $U(E)$ of $E$ is isomorphic to the tensor algebra $T(V)$ over $V$.

6. Comtrans algebras and quantum mechanics. Let $E$ be a real vector space of Hermitian operators on a complex vector space, for example the space of observables on the state space of a quantum mechanical system. Let $\hbar$ be a real number, for example Planck's constant in suitable energy $\times$ time units. The real space $E$ is a Lie algebra under the normalised binary commutator

$$
\{x, y\}=i(x y-y x) / \hbar
$$

and a Jordan algebra under the Jordan product

$$
x \cdot y=(x y+y x) / 2 .
$$

Saizew $[9, \S 2.2]$ points out the identities

$$
\hbar^{2}\{y,\{x, z\}\}=x \cdot(y \cdot z)-(x \cdot y) \cdot z
$$

and

$$
\{x \cdot y, z\}=x \cdot\{y, z\}+y \cdot\{x, z\}
$$

connecting the two operations, and regards $E$ as a "Lie--Jordan" algebra under them. The Lie--Jordan algebra structure is a useful framework for describing the correspondence principle. However, it is non-uniform in the sense that the Lie structure is infinitesimal, while the Jordan structure is not. In order to comprise the Lie and Jordan structures infinitesimally, one may instead introduce a comtrans algebra on $E$ with commutator

$$
[x, y, z]=2 x \cdot(y \cdot z)-2 y \cdot(z \cdot x)+\hbar\{x, y\} \cdot z
$$

and translator

$$
\langle x, y, z\rangle=2 z \cdot(x \cdot y)-2 y \cdot(z \cdot x)+\hbar\{x, y \cdot z\} .
$$

This comtrans algebra has a number of interesting features that are worthy of further exploration. The transposed algebra is again given by (6.5) and (6.6), except that $\hbar$ is replaced by $-\hbar$. Physically, this corresponds to reversing the direction of time [2]. The arbitrariness in the initial choice of sign for $\hbar$ is absorbed by the universal-algebraic term equivalence between $E$ and $E^{\tau}$.

The symmetric part of $E$ describes physical relationships that are time-invariant, for example the commutation relations for angular momentum operators in $\mathbb{R}^{3}$ (e.g. [8, App. B]) that correspond to the vector triple product comtrans algebra (3.3). Indeed, for a non-zero vector $\underline{v}$ in $\mathbb{R}^{3}$, let $v$ denote $\underline{v} /|\underline{v}|$ times the angular momentum operator about an axis in the direction of $\underline{v}$. By [8, B.1], linearity, and (6.1), one has

$$
\{x, y\}=-x \times y
$$


for vectors $\underline{x}, y$ in $\mathbb{R}^{3}$. Then by (4.3), (6.3) and (6.7), the commutator in the symmetric part of $E$ is

$$
2 \hbar^{2}\{\{x, y\}, z\}=2 \hbar^{2}((x \times y) \times z) .
$$

On the other hand, the antisymmetric part of $E$ describes physical relationships that are directly time-dependent. For example, let $H$ be the Hamiltonian operator of a quantum system. Then the Heisenberg equation (e.g. [8, (13.35)]) may be written in the form

$$
\frac{d}{d t}=L(1, H / \hbar)
$$

using the comtrans algebra representation notation of (5.3). Let $A(t)$ denote the operator corresponding to the observable $A$ at time $t$. Then its evolution is described by

$$
A(t)=A(0) \sum_{n=0}^{\infty} \frac{t^{n}}{n !} L(1, H / \hbar)^{n} .
$$

In other words, the evolution from $A(0)$ to $A(t)$ corresponds to applying the element

$$
\sum_{n=0}^{\infty} \frac{t^{n}}{n !} L(1, H / \hbar)^{n}
$$

from the universal enveloping algebra $U(E)$ of the comtrans algebra $E$.

\section{References}

[1] M. A. Akivis, Local algebras on a multidimensional three-web, Sibirsk. Mat. Zh. 17 (1976), 5-11 (in Russian); English translation: Siberian Math. J. 17 (1976), 3-8.

[2] I. Białynicki-Birula, A new approach to time reflection, Bull. Acad. Polon. Sci. Cl. III 5 (1957), 805-807.

[3] O. Chein, H. O. Pflugfelder and J. D. H. Smith (eds.), Quasigroups and Loops: Theory and Applications, Heldermann, Berlin 1990.

[4] V. V. Goldberg, Theory of Multicodimensional $(n+1)$-webs, Kluwer, Dordrecht 1988.

[5] H. Herrlich and G. E. Strecker, Category Theory, Allyn and Bacon, Boston 1973.

[6] K. H. Hofmann and K. Strambach, Lie's fundamental theorems for local analytical loops, Pacific J. Math. 123 (1986), 301-327.

[7] B. Huppert, Endliche Gruppen I, Springer, Berlin 1967.

[8] P. T. Matthews, Introduction to Quantum Mechanics, McGraw-Hill, New York 1963; Polish translation: PWN, Warszawa 1977.

[9] G. A. Saizew, Algebraic Problems of Mathematical and Theoretical Physics, Nauka, Moscow 1974 (in Russian); German translation: Akademie-Verlag, Berlin 1979.

[10] C. Scheiderer, Gewebegeometrie 10.6 bis 16.6.1984, Tagungsbericht 27/1984, Mathematisches Forschungsinstitut Oberwolfach, 1984.

[11] X. R. Shen and J. D. H. Smith, Simple multilinear algebras, rectangular matrices and Lie algebras, J. Algebra, to appear.

[12] -, -, Comtrans algebras and bilinear forms, Arch. Math. (Basel) 59 (1992), 327-333. 
[13] X. R. Shen and J. D. H. Smith, Representation theory of comtrans algebras, J. Pure Appl. Algebra 80 (1992), 177-195.

[14] J. D. H. Smith, Mal'cev Varieties, Springer, Berlin 1976.

[15] —, Multilinear algebras and Lie's Theorem for formal n-loops, Arch. Math. (Basel) 51 (1988), 169-177.

[16] Á. Szendrei, Clones in Universal Algebra, Les Presses de l'Université de Montréal, Montréal 1986. 